\title{
SOME OSTROWSKI TYPE INEQUALITIES FOR $n$-TIME DIFFERENTIABLE MAPPINGS AND APPLICATIONS
}

\begin{abstract}
Some generalizations of the Ostrowski inequality for $n$-time differentiable mappings are given. Applications in Numerical Integration and for power series expansions are also presented.
\end{abstract}

\section{Introduction}

In 1938, Ostrowski (see for example [3, p. 468]) proved the following integral inequality.

Let $f: I \subseteq \mathbb{R} \rightarrow \mathbb{R}$ be a differentiable mapping on $I^{\circ}\left(I^{\circ}\right.$ is the interior of $I$ ), and let $a, b \in I^{\circ}$ with $a<b$. If $f^{\prime}:(a, b) \rightarrow \mathbb{R}$ is bounded on $(a, b)$, i.e., $\left\|f^{\prime}\right\|_{\infty}:=\sup _{t \in(a, b)}\left|f^{\prime}(t)\right|<\infty$, then we have the inequality:

$$
\left|f(x)-\frac{1}{b-a} \int_{a}^{b} f(t) d t\right| \leq\left[\frac{1}{4}+\frac{\left(x-\frac{a+b}{2}\right)^{2}}{(b-a)^{2}}\right](b-a)\left\|f^{\prime}\right\|_{\infty}
$$

for all $x \in[a, b]$.

The constant $\frac{1}{4}$ is sharp in the sense that it can not be replaced by a smaller one.

For applications of Ostrowski's inequality to some special means and some numerical quadrature rules, we refer the reader to the recent paper [2] by S.S. Dragomir and S. Wang.

In 1976, G.V. Milovanović and J.E. Pečarić (see for example [3, p. 468]), proved the following generalization of Ostrowski's result:

Let $f:[a, b] \rightarrow R$ be an $n$-times differentiable function, $n \geq 1$, and such that $\left\|f^{(n)}\right\|_{\infty}:=\sup _{t \in(a, b)}\left|f^{(n)}(t)\right|<\infty$. Then

1991 Mathematics Subject Classification: Primary 26D15; Secondary 41A55.

Key words and phrases: Ostrowski Integral Inequality, Numerical Integration, Taylor's Expansions. 


$$
\begin{gathered}
\mid \frac{1}{n}\left(f(x)+\sum_{k=1}^{n-1} \frac{n-k}{k !} \cdot \frac{f^{(k-1)}(a)(x-a)^{k}-f^{(k-1)}(b)(x-b)^{k}}{b-a}\right) \\
\leq \frac{\left\|f^{(n)}\right\|_{\infty}}{n(n+1) !} \cdot \frac{(x-a)^{n+1}+(b-x)^{n+1}}{b-a}
\end{gathered}
$$

for all $x \in[a, b]$.

In [1], P. Cerone, S.S. Dragomir and J. Roumeliotis proved the following Ostrowski type inequality for twice differentiable mappings:

Let $f:[a, b] \rightarrow \mathbb{R}$ be a twice differentiable mapping on $(a, b)$ and $f^{\prime \prime}$ : $(a, b) \rightarrow \mathbb{R}$ is bounded, i.e., $\left\|f^{\prime \prime}\right\|_{\infty}=\sup _{t \in(a, b)}\left|f^{\prime \prime}(t)\right|<\infty$. Then we have the inequality:

$$
\begin{aligned}
\left|f(x)-\frac{1}{b-a} \int_{a}^{b} f(t) d t-\left(x-\frac{a+b}{2}\right) f^{\prime}(x)\right| \\
\quad \leq\left[\frac{1}{24}(b-a)^{2}+\frac{1}{2}\left(x-\frac{a+b}{2}\right)^{2}\right]\left\|f^{\prime \prime}\right\|_{\infty} \leq \frac{(b-a)^{2}}{6}\left\|f^{\prime \prime}\right\|_{\infty}
\end{aligned}
$$

for all $x \in[a, b]$.

In this paper we establish another generalization of the Ostrowski inequality for $n$-time differentiable mappings involving $\|\cdot\|_{\infty}$ which naturally generalizes the result from [1] and apply it in Numerical Integration and for power series expansions of functions on an interval. Work considering different norms is going to be presented separately, given the extent of the current development.

\section{Integral identities}

The following lemma holds (see also [4] for related results):

LEMMA 2.1. Let $f:[a, b] \rightarrow \mathbb{R}$ be a mapping such that $f^{(n-1)}$ is absolutely continuous on $[a, b]$. Then for all $x \in[a, b]$ we have the identity:

$$
\begin{aligned}
\int_{a}^{b} f(t) d t= & \sum_{k=0}^{n-1}\left[\frac{(b-x)^{k+1}+(-1)^{k}(x-a)^{k+1}}{(k+1) !}\right] f^{(k)}(x) \\
& +(-1)^{n} \int_{a}^{b} K_{n}(x, t) f^{(n)}(t) d t
\end{aligned}
$$


where the kernel $K_{n}:[a, b]^{2} \rightarrow \mathbb{R}$ is given by

$$
K_{n}(x, t):=\left\{\begin{array}{ll}
\frac{(t-a)^{n}}{n !} & \text { if } t \in[a, x] \\
\frac{(t-b)^{n}}{n !} & \text { if } t \in(x, b]
\end{array}, \quad x \in[a, b]\right.
$$

and $n$ is a natural number, $n \geq 1$.

Proof. The proof is by mathematical induction.

For $n=1$, we have to prove the equality

$$
\int_{a}^{b} f(t) d t=(b-a) f(x)-\int_{a}^{b} K_{1}(x, t) f^{(1)}(t) d t
$$

where

$$
K_{1}(x, t):= \begin{cases}t-a & \text { if } t \in[a, x] \\ t-b & \text { if } t \in(x, b]\end{cases}
$$

Integrating by parts, we have:

$$
\begin{aligned}
& \int_{a}^{b} K_{1}(x, t) f^{(1)}(t) d t \\
& \quad=\int_{a}^{x}(t-a) f^{\prime}(t) d t+\int_{x}^{b}(t-b) f^{\prime}(t) d t \\
& \quad=\left.(t-a) f(t)\right|_{a} ^{x}-\int_{a}^{x} f(t) d t+\left.(t-b) f(t)\right|_{x} ^{b}-\int_{x}^{b} f(t) d t \\
& =(x-a) f(x)+(b-x) f(x)-\int_{a}^{b} f(t) d t=(b-a) f(x)-\int_{a}^{b} f(t) d t
\end{aligned}
$$

and the identity (2.3) is proved.

Assume that (2.1) holds for " $n$ " and let us prove it for " $n+1$ ". That is, we have to prove the equality

$$
\begin{aligned}
\int_{a}^{b} f(t) d t= & \sum_{k=0}^{n}\left[\frac{(b-x)^{k+1}+(-1)^{k}(x-a)^{k+1}}{(k+1) !}\right] f^{(k)}(x) \\
& +(-1)^{n+1} \int_{a}^{b} K_{n+1}(x, t) f^{(n+1)}(t) d t
\end{aligned}
$$


where, obviously,

$$
K_{n+1}(x, t):= \begin{cases}\frac{(t-a)^{n+1}}{(n+1) !} & \text { if } t \in[a, x] \\ \frac{(t-b)^{n+1}}{(n+1) !} & \text { if } t \in(x, b]\end{cases}
$$

We have

$$
\int_{a}^{b} K_{n+1}(x, t) f^{(n+1)}(t) d t=\int_{a}^{x} \frac{(t-a)^{n+1}}{(n+1) !} f^{(n+1)}(t) d t+\int_{x}^{b} \frac{(t-b)^{n+1}}{(n+1) !} f^{(n+1)}(t) d t
$$

and integrating by parts gives

$$
\begin{aligned}
& \int_{a}^{b} K_{n+1}(x, t) f^{(n+1)}(t) d t \\
& \quad=\frac{(x-a)^{n+1}+(-1)^{n+2}(b-x)^{n+1}}{(n+1) !} f^{(n)}(x)-\int_{a}^{b} K_{n}(x, t) f^{(n)}(t) d t .
\end{aligned}
$$

That is

$$
\begin{aligned}
\int_{a}^{b} K_{n}(x, t) f^{(n)}(t) d t= & \frac{(x-a)^{n+1}+(-1)^{n+2}(b-x)^{n+1}}{(n+1) !} f^{(n)}(x) \\
& -\int_{a}^{b} K_{n+1}(x, t) f^{(n+1)}(t) d t .
\end{aligned}
$$

Now, using the mathematical induction hypothesis, we get

$$
\begin{aligned}
\int_{a}^{b} f(t) d t= & \sum_{k=0}^{n-1}\left[\frac{(b-x)^{k+1}+(-1)^{k}(x-a)^{k+1}}{(k+1) !}\right] f^{(k)}(x) \\
& +\frac{(b-x)^{n+1}+(-1)^{n}(x-a)^{n+1}}{(n+1) !} f^{(n)}(x) \\
& -(-1)^{n} \int_{a}^{b} K_{n+1}(x, t) f^{(n+1)}(t) d t \\
= & \sum_{k=0}^{n}\left[\frac{(b-x)^{k+1}+(-1)^{k}(x-a)^{k+1}}{(k+1) !}\right] f^{(k)}(x) \\
& +(-1)^{n+1} \int_{a}^{b} K_{n+1}(x, t) f^{(n+1)}(t) d t .
\end{aligned}
$$

That is, the identity (2.4); and the lemma is thus proved. 
CoROLlaRY 2.2. With the above assumptions, we have the representation

$$
\begin{aligned}
\int_{a}^{b} f(t) d t= & \sum_{k=0}^{n-1}\left[\frac{1+(-1)^{k}}{(k+1) !}\right] \frac{(b-a)^{k+1}}{2^{k+1}} f^{(k)}\left(\frac{a+b}{2}\right) \\
& +(-1)^{n} \int_{a}^{b} M_{n}(t) f^{(n)}(t) d t
\end{aligned}
$$

where

$$
M_{n}(t):=\left\{\begin{array}{ll}
\frac{(t-a)^{n}}{n !} & \text { if } t \in\left[a, \frac{a+b}{2}\right] \\
\frac{(t-b)^{n}}{n !} & \text { if } t \in\left(\frac{a+b}{2}, b\right] .
\end{array} .\right.
$$

The proof follows by Lemma 2.1 by choosing $x=\frac{a+b}{2}$.

COROLLARY 2.3. With the above assumptions, we have the representation:

$$
\begin{aligned}
\int_{a}^{b} f(t) d t= & \sum_{k=0}^{n-1} \frac{(b-a)^{k+1}}{(k+1) !}\left[\frac{f^{(k)}(a)+(-1)^{k} f^{(k)}(b)}{2}\right] \\
& +\int_{a}^{b} T_{n}(t) f^{(n)}(t) d t
\end{aligned}
$$

where

$$
T_{n}(t):=\frac{1}{n !}\left[\frac{(b-t)^{n}+(-1)^{n}(t-a)^{n}}{2}\right], \quad t \in[a, b] .
$$

Proof. In (2.1), choose firstly $x=a$ and then $x=b$. Summing the resulting two identities and then dividing by 2 gives the desired result (2.6).

Hence, the corollary is proved.

The following Taylor-like formula with integral remainder also holds:

COROLlARY 2.4. Let $g:[a, y] \rightarrow \mathbb{R}$ be a mapping such that $g^{(n)}$ is absolutely continuous on $[a, y]$. Then for all $x \in[a, y]$, we have the identity

$$
\begin{aligned}
g(y)= & g(a)+\sum_{k=0}^{n-1} \frac{\left[(y-x)^{k+1}+(-1)^{k}(x-a)^{k+1}\right]}{(k+1) !} g^{(k+1)}(x) \\
& +(-1)^{n} \int_{a}^{y} K \widetilde{K}_{n}(x, t) g^{(n+1)}(t) d t .
\end{aligned}
$$

The proof is obvious by Lemma 2.1 choosing $f=g^{\prime}, b=y$ and $\widetilde{K} \equiv K$ with $b=y$.

REMARK 2.1. If we choose $n=1$ in (2.1), we get the identity

$$
\int_{a}^{b} f(t) d t=(b-a) f(x)-\int_{a}^{b} K_{1}(x, t) f^{\prime}(t) d t
$$


for all $x \in[a, b]$, where

$$
K_{1}(x, t):= \begin{cases}t-a & \text { if } t \in[a, x] \\ t-b & \text { if } t \in(x, b]\end{cases}
$$

which is the identity employed by S.S. Dragomir and S. Wang to prove an Ostrowski type inequality in paper [2].

If in (2.5) we choose $n=1$, then we get

$$
\int_{a}^{b} f(t) d t=(b-a) f\left(\frac{a+b}{2}\right)-\int_{a}^{b} M_{1}(t) f^{\prime}(t) d t
$$

where

$$
M_{1}(t)= \begin{cases}t-a & \text { if } t \in\left[a, \frac{a+b}{2}\right] \\ t-b & \text { if } t \in\left(\frac{a+b}{2}, b\right]\end{cases}
$$

which gives the mid-point type inequality useful in Numerical Analysis.

Also, if we put $n=1$ in (2.6), we get the trapezoid identity

$$
\int_{a}^{b} f(t) d t=\frac{f(a)+f(b)}{2}(b-a)+\int_{a}^{b} T_{1}(t) f^{\prime}(t) d t
$$

where

$$
T_{1}(t)=\frac{a+b}{2}-t, \quad t \in[a, b] .
$$

Finally, if in the Taylor-like formula (2.7) we put $n=1$, we get

$$
g(y)=g(a)+(y-a) g^{\prime}(x)-\int_{a}^{y} \widetilde{K}_{1}(x, t) g^{(2)}(t) d t
$$

where $x \in[a, y]$.

REMARK 2.2. If we choose $n=2$ in (2.1), we get the identity:

(2.11) $\int_{a}^{b} f(t) d t=(b-a) f(x)-\left(x-\frac{a+b}{2}\right) f^{\prime}(x)+\int_{a}^{b} K_{2}(x, t) f^{\prime \prime}(t) d t$,

where

$$
K_{2}(x, t):= \begin{cases}\frac{(t-a)^{2}}{2} & \text { if } t \in[a, x] \\ \frac{(t-b)^{2}}{2} & \text { if } t \in(x, b]\end{cases}
$$

and $x \in[a, b]$, which is the identity employed by P. Cerone, S.S. Dragomir and $J$. Roumeliotis to prove some Ostrowski type inequalities for twice differentiable mappings in the paper [1]. 
If in (2.5) we choose $n=2$, then we get

$$
\int_{a}^{b} f(t) d t=(b-a) f\left(\frac{a+b}{2}\right)+\int_{a}^{b} M_{2}(t) f^{\prime \prime}(t) d t,
$$

where

$$
M_{2}(t)= \begin{cases}\frac{(t-a)^{2}}{2} & \text { if } t \in\left[a, \frac{a+b}{2}\right] \\ \frac{(t-b)^{2}}{2} & \text { if } t \in\left(\frac{a+b}{2}, b\right]\end{cases}
$$

which is the classical mid-point identity.

Also, if we put $n=2$ in (2.6), we get the identity

$$
\begin{aligned}
\int_{a}^{b} f(t) d t= & \frac{f(a)+f(b)}{2}(b-a)+\frac{(b-a)^{2}}{2} \cdot \frac{f^{\prime}(a)-f^{\prime}(b)}{2} \\
& +\int_{a}^{b} T_{2}(t) f^{\prime \prime}(t) d t,
\end{aligned}
$$

where

$$
T_{2}(t)=\frac{1}{2} \cdot \frac{(b-t)^{2}+(t-a)^{2}}{2}, \quad t \in[a, b] .
$$

Finally, if we put $n=2$ in (2.7), we get

$$
\begin{aligned}
g(y)= & g(a)+(y-a) g^{\prime}(x)-(y-a)\left(x-\frac{a+y}{2}\right) g^{\prime \prime}(x) \\
& +\int_{a}^{y} K_{2}(y, t) g^{(3)}(t) d t
\end{aligned}
$$

where $K_{2}$ is as above and $a \leq x \leq y$.

3. Some integral inequalities for $\|\cdot\|_{\infty}-$ norm

The following theorem holds:

THEOREM 3.1. Let $f:[a, b] \rightarrow \mathbb{R}$ be a mapping such that $f^{(n-1)}$ is absolutely continuous on $[a, b]$ and $f^{(n)} \in L_{\infty}[a, b]$. Then for all $x \in[a, b]$, we have the inequality:

$$
\begin{aligned}
& \left|\int_{a}^{b} f(t) d t-\sum_{k=0}^{n-1}\left[\frac{(b-x)^{k+1}+(-1)^{k}(x-a)^{k+1}}{(k+1) !}\right] f^{(k)}(x)\right| \\
& \leq \frac{\left\|f^{(n)}\right\|_{\infty}}{(n+1) !}\left[(x-a)^{n+1}+(b-x)^{n+1}\right] \leq \frac{\left\|f^{(n)}\right\|_{\infty}(b-a)^{n+1}}{(n+1) !}
\end{aligned}
$$

where

$$
\left\|f^{(n)}\right\|_{\infty}:=\sup _{t \in[a, b]}\left|f^{(n)}(t)\right|<\infty .
$$

The inequalities are sharp and the constant 1 is the best possible. 
Proof. Using the identity (2.1), we have:

$$
\begin{aligned}
\mid \int_{a}^{b} f(t) d t & -\sum_{k=0}^{n-1}\left[\frac{(b-x)^{k+1}+(-1)^{k}(x-a)^{k+1}}{(k+1) !}\right] f^{(k)}(x) \mid \\
& =\left|\int_{a}^{b} K_{n}(x, t) f^{(n)}(t) d t\right| \leq\left\|f^{(n)}\right\|_{\infty} \int_{a}^{b}\left|K_{n}(x, t)\right| d t, \\
& =\left\|f^{(n)}\right\|_{\infty}\left[\int_{a}^{x} \frac{(t-a)^{n}}{n !} d t+\int_{x}^{b} \frac{(b-t)^{n}}{n !} d t\right] \\
& =\frac{\left\|f^{(n)}\right\|_{\infty}}{(n+1) !}\left[(x-a)^{n+1}+(b-x)^{n+1}\right],
\end{aligned}
$$

and the first part of inequality (3.1) is proved.

To prove the second inequality in (3.1), we observe that

$$
(x-a)^{n+1}+(b-x)^{n+1} \leq(b-a)^{n+1},
$$

for all $x \in[a, b]$.

Now we are going to deal with the sharpness of the inequality (3.1).

Consider $f:[a, b] \rightarrow \mathbb{R}$ to be such that

$$
f(t)=\frac{\left(t-\frac{a+b}{2}\right)^{n}}{n !}
$$

Thus,

$$
f^{(k)}(x)=\frac{\left(x-\frac{a+b}{2}\right)^{n-k}}{(n-k) !}, \quad\left\|f^{(n)}\right\|_{\infty}=1
$$

and

$$
\int_{a}^{b} \frac{\left(t-\frac{a+b}{2}\right)^{n}}{n !} d t=\frac{2}{(n+1) !}\left(\frac{b-a}{2}\right)^{n+1} .
$$

Therefore, from (3.1),

$$
\begin{aligned}
\left.\mid \frac{2}{(n+1) !}\left(\frac{b-a}{2}\right)^{n+1}-\sum_{k=0}^{n-1} \frac{\left[(b-x)^{k+1}\right.}{}+(-1)^{k}(x-a)^{k+1}\right] & \frac{\left(x-\frac{a+b}{2}\right)^{n-k}}{(n-k) !} \mid \\
& \leq \frac{C}{(n+1) !}\left[(x-a)^{n+1}+(b-x)^{n+1}\right]
\end{aligned}
$$

Taking $x=\frac{a+b}{2}$ then gives

$$
\left|\frac{2}{(n+1) !}\left(\frac{b-a}{2}\right)^{n+1}\right| \leq \frac{C}{(n+1) !} 2\left(\frac{b-a}{2}\right)^{n+1},
$$

and so $1 \leq C$ since $b>a$ and thus $C=1$ is the best constant. Hence the theorem is completely proved. 
Taking into account the fact that the mapping $h_{n}:[a, b] \rightarrow \mathbb{R}, h_{n}(x):=$ $x-a)^{n+1}+(b-x)^{n+1}$, has the property

$$
\inf _{x \in[a, b]} h_{n}(x)=h_{n}\left(\frac{a+b}{2}\right)=\frac{(b-a)^{n+1}}{2^{n}}
$$

then the best inequality we can get from (3.1) is the one for which $x=\frac{a+b}{2}$.

In this way, we can state the following corollary.

Corollary 3.2. Assume that $f$ is as in Theorem 3.1. Then we have the inequality:

$$
\begin{gathered}
\left|\int_{a}^{b} f(t) d t-\sum_{k=0}^{n-1}\left[\frac{1+(-1)^{k}}{(k+1) !}\right] \frac{(b-a)^{k+1}}{2^{k+1}} f^{(k)}\left(\frac{a+b}{2}\right)\right| \\
\leq \frac{\left\|f^{(n)}\right\|_{\infty}(b-a)^{n+1}}{2^{n}(n+1) !}
\end{gathered}
$$

Another result which generalizes the trapezoid inequality, is the following one:

CoROLlary 3.3. With the above assumptions, we have the inequality:

$$
\begin{aligned}
& \left|\int_{a}^{b} f(t) d t-\sum_{k=0}^{n-1} \frac{(b-a)^{k+1}}{(k+1) !}\left[\frac{f^{(k)}(a)+(-1)^{k} f^{(k)}(b)}{2}\right]\right| \\
& \leq \frac{(b-a)^{n+1}}{(n+1) !}\left\|f^{(n)}\right\|_{\infty} \times\left\{\begin{array}{ll}
1 & \text { if } n=2 r \\
\frac{2^{2 r+1}-1}{2^{2 r}} & \text { if } n=2 r+1
\end{array} .\right.
\end{aligned}
$$

Proof. Using the identity (2.6), we get

$$
\begin{aligned}
\mid \int_{a}^{b} f(t) d t-\sum_{k=0}^{n-1} \frac{(b-a)^{k+1}}{(k+1) !} & {\left[\frac{f^{(k)}(a)+(-1)^{k} f^{(k)}(b)}{2}\right] \mid } \\
& =\left|\int_{a}^{b} T_{n}(t) f^{(n)}(t) d t\right| \leq\left\|f^{(n)}\right\|_{\infty} \int_{a}^{b}\left|T_{n}(t)\right| d t .
\end{aligned}
$$

If $n=2 r$, then

$$
\begin{aligned}
\int_{a}^{b}\left|T_{n}(t)\right| d t & =\frac{1}{(2 r) !} \int_{a}^{b}\left[\frac{(b-t)^{2 r}+(t-a)^{2 r}}{2}\right] d t \\
& =\frac{1}{(2 r) !} \cdot \frac{1}{2}\left[\frac{(b-a)^{2 r+1}}{(2 r+1)}+\frac{(b-a)^{2 r+1}}{(2 r+1)}\right]=\frac{(b-a)^{2 r+1}}{(2 r+1) !}
\end{aligned}
$$

For $n=2 r+1$, put $h_{2 r+1}(t):=(b-t)^{2 r+1}-(t-a)^{2 r+1}, t \in[a, b]$. Observe that $h_{2 r+1}(t)=0$ iff $t=\frac{a+b}{2}$ and $h_{2 r+1}(t)>0$ if $t \in\left[a, \frac{a+b}{2}\right)$ and $h_{2 r+1}(t)<0$ if $t \in\left(\frac{a+b}{2}, b\right]$. 
Then

$$
\begin{aligned}
\int_{a}^{b}\left|T_{2 r+1}(t)\right| d t= & \int_{a}^{\frac{a+b}{2}}\left[(b-t)^{2 r+1}-(t-a)^{2 r+1}\right] d t \\
& +\int_{\frac{a+b}{2}}^{b}\left[(t-a)^{2 r+1}-(b-t)^{2 r+1}\right] d t \\
= & 2 \frac{(b-a)^{2 r+2}}{2 r+2}-\frac{4\left(\frac{b-a}{2}\right)^{2 r+2}}{2 r+2} \\
= & \frac{1}{2 r+2}\left[2(b-a)^{2 r+2}-\frac{(b-a)^{2 r+2}}{2^{2 r}}\right] \\
= & \frac{(b-a)^{2 r+2}}{2 r+2}\left(2-\frac{1}{2^{2 r}}\right)=\frac{(b-a)^{2 r+2}}{2 r+2} \cdot \frac{2^{2 r+1}-1}{2^{2 r}} .
\end{aligned}
$$

Using (3.4) we get the desired inequality (3.3).

The following inequality in terms of $\|\cdot\|_{\infty}-$ norm for the Taylor like expansion (2.7) also holds:

Corollary 3.4. Let $g$ be as in Corollary 2.4. Then we have the inequality:

$$
\begin{aligned}
& \left|g(y)-g(a)-\sum_{k=0}^{n-1} \frac{\left[(y-x)^{k+1}+(-1)^{k}(x-a)^{k+1}\right]}{(k+1) !} g^{(k+1)}(x)\right| \\
& \quad \leq \frac{\left\|g^{(n+1)}\right\|_{\infty}}{(n+1) !}\left[(y-x)^{n+1}+(x-a)^{n+1}\right] \leq \frac{\left\|g^{(n+1)}\right\|_{\infty}}{(n+1) !}(y-a)^{n+1}
\end{aligned}
$$

for all $a \leq x \leq y$.

REMARK 3.1. It is well known that for the classical Taylor expansion around a we have the inequality

$$
\left|g(y)-\sum_{k=0}^{n} \frac{(y-a)^{k}}{k !} g^{(k)}(a)\right| \leq \frac{\left\|g^{(n+1)}\right\|_{\infty}}{(n+1) !}(y-a)^{n+1}
$$

for all $y \geq a$. It is clear now that the above approximation (3.6) around the arbitrary point $x \in[a, y]$ provides a better approximation for the mapping $g$ at the point $y$ than the classical Taylor expansion around the point $a$.

If in (3.6) we choose $x=\frac{a+y}{2}$, then we get

$$
\begin{gathered}
\left|g(y)-g(a)-\sum_{k=1}^{n} \frac{\left[1+(-1)^{k-1}\right]}{k !} \frac{(y-a)^{k}}{2^{k}} g^{(k)}\left(\frac{a+y}{2}\right)\right| \\
\leq \frac{\left\|g^{(n+1)}\right\|_{\infty}}{(n+1) ! 2^{n}}(y-a)^{n+1}
\end{gathered}
$$


The above inequality (3.8) shows that for $g \in C^{\infty}[a, b]$ the series

$$
g(a)+\sum_{k=0}^{\infty} \frac{\left[1+(-1)^{k}\right]}{(k+1) !} \frac{(y-a)^{k+1}}{2^{k+1}} g^{(k+1)}\left(\frac{a+y}{2}\right)
$$

converges more rapidly to $g(y)$ than the usual one

$$
\sum_{k=0}^{\infty} \frac{(y-a)^{k} g^{(k)}(a)}{k !}
$$

which comes from Taylor's expansion. Further, it should be noted that (3.8) only involves odd derivatives of $g$.

REMARK 3.2. If in the inequality (3.1) we choose $n=1$ we get

$$
\left|\int_{a}^{b} f(t) d t-(b-a) f(x)\right| \leq \frac{(x-a)^{2}+(b-x)^{2}}{2}\left\|f^{\prime}\right\|_{\infty} .
$$

A simple calculation shows that

$$
\frac{1}{2}\left[(x-a)^{2}+(b-x)^{2}\right]=\frac{1}{4}(b-a)^{2}+\left(x-\frac{a+b}{2}\right)^{2}
$$

and consequently we obtain the Ostrowski inequality:

$$
\left|\int_{a}^{b} f(t) d t-(b-a) f(x)\right| \leq\left[\frac{1}{4}+\frac{\left(x-\frac{a+b}{2}\right)^{2}}{(b-a)^{2}}\right](b-a)^{2}\left\|f^{\prime}\right\|_{\infty} .
$$

for all $x \in[a, b]$.

If in (3.2) we put $n=1$, we get the mid-point inequality

$$
\left|\int_{a}^{b} f(t) d t-f\left(\frac{a+b}{2}\right)(b-a)\right| \leq \frac{1}{4}(b-a)^{2}\left\|f^{\prime}\right\|_{\infty} .
$$

From the inequality (3.4), for $n=1$, we get the trapezoid inequality

$$
\left|\int_{a}^{b} f(t) d t-\frac{f(a)+f(b)}{2}(b-a)\right| \leq \frac{1}{2}(b-a)^{2}\left\|f^{\prime}\right\|_{\infty} .
$$

Also, from (3.6) we deduce

$$
\left|g(y)-g(a)-(y-a) g^{\prime}(x)\right| \leq\left[\frac{1}{4}+\frac{\left(x-\frac{a+y}{2}\right)^{2}}{(y-a)^{2}}\right]\left\|g^{\prime \prime}\right\|_{\infty}
$$

for all $a \leq x \leq y$.

REMARK 3.3. If in the inequality (3.1) we choose $n=2$, then we get

$\left|\int_{a}^{b} f(t) d t-(b-a) f(x)+\left(x-\frac{a+b}{2}\right)(b-a) f^{\prime}(x)\right| \leq \frac{1}{6}\left[(x-a)^{3}+(b-x)^{3}\right]\left\|f^{\prime \prime}\right\|_{\alpha}$ for all $x \in[a, b]$. 
Now, observe that

$$
(x-a)^{3}+(b-x)^{3}=(b-a)\left[\left(\frac{b-a}{2}\right)^{2}+3\left(x-\frac{a+b}{2}\right)^{2}\right]
$$

and then we recapture the result obtained in [1], namely

$$
\begin{aligned}
\mid \int_{a}^{b} f(t) d t-(b-a) f(x)+ & \left(x-\frac{a+b}{2}\right)(b-a) f^{\prime}(x) \mid \\
& \leq\left[\frac{1}{24}+\frac{1}{2} \frac{\left(x-\frac{a+b}{2}\right)^{2}}{(b-a)^{2}}\right](b-a)^{3}\left\|f^{\prime \prime}\right\|_{\infty} .
\end{aligned}
$$

If we put $n=2$ in (3.3), we get the classical mid-point inequality

$$
\left|\int_{a}^{b} f(t) d t-(b-a) f\left(\frac{a+b}{2}\right)\right| \leq \frac{1}{24}(b-a)^{3}\left\|f^{\prime \prime}\right\|_{\infty} .
$$

Now, if in (3.4) we put $n=2$, then we get the inequality:

$$
\begin{gathered}
\left|\int_{a}^{b} f(t) d t-\frac{f(a)+f(b)}{2}(b-a)-\frac{(b-a)^{2}}{2} \cdot \frac{f^{\prime}(a)-f^{\prime}(b)}{2}\right| \\
\leq \frac{(b-a)^{3}}{6}\left\|f^{\prime \prime}\right\|_{\infty} .
\end{gathered}
$$

Finally, if we put $n=2$ in (3.6), then we get the inequality:

$$
\begin{aligned}
\mid g(y)-g(a)-(y-a) g^{\prime}(x)+ & (y-a)\left(x-\frac{a+y}{2}\right) g^{\prime \prime}(x) \mid \\
& \leq\left[\frac{1}{24}+\frac{1}{2} \cdot \frac{\left(x-\frac{a+y}{2}\right)^{2}}{(y-a)^{2}}\right](y-a)^{3}\left\|g^{\prime \prime \prime}\right\|_{\infty} .
\end{aligned}
$$

\section{Applications for numerical integration}

Consider the partition $I_{m}: a=x_{0}<x_{1}<\ldots<x_{m-1}<x_{m}=b$ of the interval $[a, b]$ and the intermediate points $\xi=\left(\xi_{0}, \ldots, \xi_{m-1}\right)$ where $\xi_{j} \in\left[x_{j}, x_{j+1}\right] j=0, \ldots, m-1$. Define the formula

$$
F_{m, k}\left(f, I_{m}, \xi\right):=\sum_{j=0}^{m-1} \sum_{k=0}^{n-1} \frac{\left[\left(x_{j+1}-\xi_{j}\right)^{k+1}+(-1)^{k}\left(\xi_{j}-x_{j}\right)^{k+1}\right]}{(k+1) !} f^{(k)}\left(\xi_{j}\right)
$$

which can be regarded as a perturbation of Riemann's sum

$$
\Gamma\left(f, I_{m}, \xi\right)=\sum_{j=0}^{m-1} f\left(\xi_{j}\right) h_{j}
$$

where $h_{j}:=x_{j+1}-x_{j}, \quad j=0, \ldots, m-1$.

The following theorem holds. 
THEOREM 4.1. Let $f:[a, b] \rightarrow \mathbb{R}$ be a mapping such that $f^{(n-1)}$ is absolutely continuous on $[a, b]$ and $I_{m} a$ partitioning of $[a, b]$ as above. Then we have the quadrature formula

$$
\int_{a}^{b} f(x) d x=F_{m, k}\left(f, I_{m}, \xi\right)+R_{m, k}\left(f, I_{m}, \xi\right)
$$

where $F_{m, k}$ is defined above and the remainder $R_{m, k}$ satisfies the estimation:

$$
\begin{aligned}
\left|R_{m, k}\left(f, I_{m}, \xi\right)\right| & \leq \frac{\left\|f^{(n)}\right\|_{\infty}}{(n+1) !} \sum_{j=0}^{m-1}\left[\left(\xi_{j}-x_{j}\right)^{n+1}+\left(x_{j+1}-\xi_{j}\right)^{n+1}\right] \\
& \leq \frac{\left\|f^{(n)}\right\|_{\infty}}{(n+1) !} \sum_{j=0}^{m-1} h_{j}^{n+1}
\end{aligned}
$$

for all $\xi$ as above.

Pr oof. Apply Theorem 3.1 on the interval $\left[x_{j}, x_{j+1}\right]$ to get

$$
\begin{aligned}
\mid \int_{x_{j}}^{x_{j}+1} f(t) d t & -\sum_{k=0}^{n-1}\left[\frac{\left[\left(x_{j+1}-\xi_{j}\right)^{k+1}+(-1)^{k}\left(\xi_{j}-x_{j}\right)^{k+1}\right]}{(k+1) !}\right] f^{(k)}\left(\xi_{j}\right) \mid \\
& \leq \frac{\left\|f^{(n)}\right\|_{\infty}}{(n+1) !}\left[\left(\xi_{j}-x_{j}\right)^{n+1}+\left(x_{j+1}-\xi_{j}\right)^{n+1}\right] \leq \frac{\left\|f^{(n)}\right\|_{\infty}}{(n+1) !} h_{j}^{n+1}
\end{aligned}
$$

for all $j \in\{0, \ldots, m-1\}$.

Summing over $j$ from 0 to $m-1$ and using the generalized triangle inequality, we deduce the desired estimation (4.17).

As an interesting particular case, we can consider the following perturbed mid-point formula

$$
M_{m, k}\left(f, I_{m}\right):=\sum_{j=0}^{m-1} \sum_{k=0}^{n-1}\left[\frac{1+(-1)^{k}}{(k+1) !}\right] \frac{h_{j}^{k+1}}{2^{k+1}} f^{(k)}\left(\frac{x_{j}+x_{j+1}}{2}\right),
$$

which in effect involves only even $k$.

We state the following result concerning the estimation of the remainder term.

COROLlaRY 4.2. Let $f$ and $I_{m}$ be as in Theorem 4.1. Then we have

$$
\int_{a}^{b} f(t) d t=M_{m, k}\left(f, I_{m}\right)+R_{m, k}\left(f, I_{m}\right)
$$

and the remainder term $R_{m, k}$ satisfies the estimation

$$
\left|R_{m, k}\left(f, I_{m}\right)\right| \leq \frac{\left\|f^{(n)}\right\|_{\infty}}{2^{n}(n+1) !} \sum_{j=0}^{m-1} h_{j}^{n+1} .
$$


We can consider the following perturbed version of the trapezoid formula:

$$
T_{m, k}\left(f, I_{m}\right):=\sum_{j=0}^{m-1} \sum_{k=0}^{n-1} \frac{h_{j}^{k+1}}{(k+1) !}\left[\frac{f^{(k)}\left(x_{j}\right)+(-1)^{k} f^{(k)}\left(x_{j+1}\right)}{2}\right] .
$$

By the use of Corollary 3.3, we have the following approximation of the integral $\int_{a}^{b} f(t) d t$ in terms of $T_{m, k}\left(f, I_{m}\right)$ :

Corollary 4.3. Let $f$ and $I_{m}$ be as in Theorem 4.1. Then we have

$$
\int_{a}^{b} f(t) d t=T_{m, k}\left(f, I_{m}\right)+\tilde{R}_{m, k}\left(f, I_{m}\right)
$$

and the remainder $\tilde{R}_{m, k}\left(f, I_{m}\right)$ satisfies the inequality

$$
\left|\tilde{R}_{m, k}\left(f, I_{m}\right)\right| \leq \frac{C_{n}}{(n+1) !}\left\|f^{(n)}\right\|_{\infty} \sum_{j=0}^{m-1} h_{j}^{n+1},
$$

where

$$
C_{n}:=\left\{\begin{array}{lr}
1 & \text { if } n=2 r \\
\frac{2^{2 r+1}-1}{2^{2 r}} & \text { if } n=2 r+1
\end{array} .\right.
$$

REMARK 4.1. a) If we choose $n=1$ in the above quadrature formulae (4.16) and (4.18), we recapture some results from the paper [2].

b) If we put $n=2$, then by the above Theorem 4.1 and Corollary 4.2, we recover some results from the paper [1].

We omit the details.

\section{Applications for some particular mappings}

a) Consider $g: \mathbb{R} \rightarrow \mathbb{R}, g(x)=e^{x}$. Then $g^{(n)}(x)=e^{x}, n \in \mathbb{N}$ and

$$
\left\|g^{(n+1)}\right\|_{\infty}=\sup _{t \in[a, y]}\left|g^{(n+1)}(t)\right|=e^{y}
$$

Using inequality (3.6), we have

$$
\begin{aligned}
& \left|e^{y}-e^{a}-e^{x} \sum_{k=0}^{n-1} \frac{\left[(y-x)^{k+1}+(-1)^{k}(x-a)^{k+1}\right]}{(k+1) !}\right| \\
& \quad \leq \frac{e^{y}}{(n+1) !}\left[(y-x)^{n+1}+(x-a)^{n+1}\right] \leq \frac{e^{y}}{(n+1) !}(y-a)^{n+1}
\end{aligned}
$$

for all $a \leq x \leq y$.

Particularly, if we choose $a=0$, then we get

$$
\left|e^{y}-1-e^{x} \sum_{k=0}^{n-1} \frac{\left[(y-x)^{k+1}+(-1)^{k} x^{k+1}\right]}{(k+1) !}\right|
$$




$$
\leq \frac{e^{y}}{(n+1) !}\left[(y-x)^{n+1}+x^{n+1}\right] \leq \frac{e^{y}}{(n+1) !} y^{n+1} .
$$

Moreover, if we choose $x=\frac{y}{2}$, then we get

$$
\left|e^{y}-1-e^{\frac{y}{2}} \sum_{k=0}^{n-1} \frac{1+(-1)^{k}}{(k+1) !} \cdot \frac{y^{k+1}}{2^{k+1}}\right| \leq \frac{e^{y} y^{n+1}}{2^{n}(n+1) !}
$$

for all $y \geq 0$.

b) Consider $g:(0, \infty) \rightarrow \mathbb{R}, g(x)=\ln x$. Then

$$
g^{(n)}(x)=\frac{(-1)^{n-1}(n-1) !}{x^{n}}, \quad n \geq 1, x>0
$$

and

$$
\left\|g^{(n+1)}\right\|_{\infty}=\sup _{t \in[a, y]}\left|\frac{(-1)^{n} n !}{t^{n+1}}\right|=\frac{n !}{a^{n+1}}, \quad a>0 .
$$

Using the inequality (3.6) we can state:

$$
\begin{aligned}
& \left|\ln y-\ln a-\sum_{k=0}^{n-1} \frac{(y-x)^{k+1}+(-1)^{k}(x-a)^{k+1}}{(k+1) !} \cdot \frac{(-1)^{k} k !}{x^{k+1}}\right| \\
& \leq \frac{n !}{(n+1) ! a^{n+1}}\left[(y-x)^{n+1}+(x-a)^{n+1}\right] \leq \frac{n !}{(n+1) ! a^{n+1}}(y-a)^{n+1}
\end{aligned}
$$

which is equivalent to

$$
\begin{gathered}
\left|\ln \left(\frac{y}{a}\right)-\sum_{k=0}^{n-1} \frac{1}{k+1} \cdot \frac{(x-a)^{k+1}+(-1)^{k}(y-x)^{k+1}}{x^{k+1}}\right| \\
\quad ; l \leq \frac{(y-x)^{n+1}+(x-a)^{n+1}}{(n+1) a^{n+1}} \leq \frac{1}{(n+1) a^{n+1}}(y-a)^{n+1} .
\end{gathered}
$$

Now, if we choose in (5.4) $y=z+1, x=w+1, a=1, z \geq w \geq 0$, then we get

$$
\begin{gathered}
\left|\ln (z+1)-\sum_{k=0}^{n-1} \frac{1}{k+1} \cdot \frac{w^{k+1}+(-1)^{k}(z-w)^{k+1}}{(w+1)^{k+1}}\right| \\
\leq \frac{(z-w)^{n+1}+w^{n+1}}{n+1} \leq \frac{1}{(n+1)} z^{n+1} .
\end{gathered}
$$

Finally, if we choose in (5.4), $y=u a, x=w a$ with $u \geq w>1$, then we have

$$
\begin{aligned}
\left|\ln u-\sum_{k=0}^{n-1} \frac{1}{k+1} \frac{(w-1)^{k+1}+(-1)^{k}(u-w)^{k+1}}{w^{k+1}}\right| \\
\leq \frac{(u-w)^{n+1}+(w-1)^{m+1}}{n+1} \leq \frac{(u-1)^{n+1}}{(n+1)} .
\end{aligned}
$$




\section{References}

[1] P. Cerone, S. S. Dragomir and J. Roumeliotis, An inequality of Ostrowski type for mappings whose second derivatives are bounded and applications, submitted.

[2] S. S. Dragomir and S. Wang, Applications of Ostrowski's inequality to the estimation of error bounds for some special means and some numerical quadrature rules, Appl. Math. Lett. 11 (1998), 105-109.

[3] D. S. Mitrinović, J. E. Pečarić and A. M. Fink, Inequalities for Functions and Their Integrals and Derivatives, Kluwer Academic, Dordrecht, 1994.

[4] J. Sándor, On the Jensen-Hadamard inequality, Studia Univ. Babes-Bolai, Math., 36 (1) (1991), 9-15.

SCHOOL OF COMMUNICATIONS AND INFORMATICS

VICTORIA UNIVERSITY OF TECHNOLOGY

PO BOX 14428

MELBOURNE CITY MC, VICTORIA 8001, AUSTRALIA

E-mail: \{pc, sever, john\}Omatilds.vu.edu.su

Received November 29, 1998. 